\title{
Distortion Risk Measures for Non-negative Multivariate Risks
}

\author{
Montserrat Guillen $₫$ 1, José María Sarabia ${ }^{b}$, \\ Jaume Belles-Sampera ${ }^{a}$ and Faustino Prieto ${ }^{b}$ \\ ${ }^{a}$ Department of Econometrics, Riskcenter-IREA, University of Barcelona, Av. \\ Diagonal, 690, 08034 Barcelona, Spain \\ ${ }^{b}$ Department of Economics, University of Cantabria, 39005 Santander, Spain
}

\begin{abstract}
We apply distortion functions to bivariate survival functions for nonnegative random variables. This leads to a natural extension of univariate distortion risk measures to the multivariate setting. For Gini's principle, the proportional hazard transform and the dual power transform distortions, certain families of multivariate distributions lead to a straightforward risk measure. We show that an exact analytical expression can be obtained in some cases. We consider the independence case, the bivariate Pareto distribution and the bivariate exponential distribution. An illustration of the estimation procedure and the interpretation is also included. In the case study we consider two loss events with one single risk value and monitor the two events together over four different periods. We conclude that the Dual Power Transform gives more weight to the observations of extreme losses, but that the distortion parameter can modulate this influence in all cases. In our example, multivariate risk clearly diminishes over time.
\end{abstract}

Key Words: Distortion functions, multivariate risk factors, multi-period risk assessment, dependence.

\section{Introduction}

Classical risk measures are defined on univariate risks, i.e. on unidimensional random variables, and not on a multivariate setting. However, risk evalua-

\footnotetext{
${ }^{1}$ Corresponding author. Tel.: +34934037039 Fax: +34934021821 E-mail address: mguillen@ub.edu
} 
tion problems in real life are rarely of dimension one. In many practical applications, it is usual to deal with multidimensionality by transforming multivariate risks into a unidimensional risk using some aggregation procedure, for instance using the sum of risks. Once the multiple dimension of the risk problem has been reduced to one dimension, then classical risk measures can be used to quantify risk.

The purpose of this paper is to propose a set of risk measures for nonnegative multivariate risks from a different perspective. Our approach to multivariate risk assessment problems differs from the traditional procedure in the way aggregation is performed. Instead of transforming the multivariate random variable first and then quantifying the risk in the univariate setting, we concentrate on the whole multidimensional distribution and we define a risk measurement one-dimensional value there. We follow the definition given by Rüschendorf (2013, p. 180) and present it in Section 2 .

Risk management often requires multivariate risk measures that capture the dependence among many risk factors. When considering all the dimensions, it is natural to take the joint multivariate distribution function of the risks as the starting point. For instance, the quantile of the joint distribution leads to the analysis of critical layers, as defined by Salvadori et al. (2011) or discussed later by Di Bernardino and Palacios-Rodríguez (2017), which are multidimensional by definition. Our approach is totally different, we aim to obtain one single value that summarizes the risk of a multivariate random vector, but we apply a distortion to the joint survival multidimensional function and then we do a multiple integration in order to obtain a summary value. The main advantage is that we do not work with vectors of risk measures, moreover, we show that for some special multivariate distributions, this approach provides simple analytic expressions. A potential drawback is that the distortion of the multivariate survival and the multiple integral, even if it is an elegant generalization, is a summary measure that combines all dimensions in one and may be difficult to interpret.

As stated in Embrechts and Puccetti (2006), in the risk management and finance literature random vectors are referred to as portfolios and the individual random sub-vectors as risks. Usually portfolios of identically distributed, non-negative risks are considered. Note that even if in finance returns can be positive or negative, the risk manager looks at losses, so that one of the two axes is the side of interest. According to Sun et al. (2017) portfolio risk management measures the distribution of losses in a portfolio over a fixed horizon, but the dependence among risk factors complicates the computation. The 
dependence structure is then assumed from a joint multivariate distribution that has a fixed dependence over time or a multivariate copula function that could include some time varying dependence. Alternatively, in order to analyse each dimension separately, one has to take the marginal distribution or the component-wise measures. Cousin and Di Bernardino (2013) dealt with multidimensionality by analysing vector-valued measures with the same dimension as the underlying risk variables, this approach is also referred to as set-valued risk measures. From the vector of risk measures these authors define the lower-orthant VaR, which constructed from level sets of multivariate distribution functions and the upperorthant $\mathrm{VaR}$ is constructed from level sets of multivariate survival functions.

We should note that an application of multivariate risk measures is found in risk management of financial institutions, since Basel III requires a minimum capital which is derived from the analysis of risk on an aggregated basis. Traditional univariate risk measures cannot address portfolio risk management as a whole.

The set of risk measures that we propose can be called distortion risk measures for non-negative multivariate risks. As it is explained along the next sections, there is a natural parallelism between unidimensional distortion risk measures introduced by Wang $(1995 \mathrm{a}, \underline{\mathrm{b}})$ ) and the risk measures introduced in this paper.

In the insurance setting and in operational risk in particular, risk managers generally look at losses only and they are positive values. If these results were to be extended to the analysis of returns, which can be either positive or negative, then one could use the same principle of distortion of the joint survival. Belles-Sampera et al. (2013) indicated that distortion risk measures can be interpreted as aggregation operators for finite random variables that do not necessarily have to be positive.

We show in the illustrations that our proposal provides a good method to monitor multivariate risks that can be specially interesting in the context of operational risk analysis. 


\section{Distortion risk measures for the non-negative univariate case}

Let us assume a probability space $(\Omega, \mathcal{A}, P)$ with sample space $\Omega$, a $\sigma$-algebra $\mathcal{A}$ and a probability $P$ from $\mathcal{A}$ to $[0,1]$, and the set of all random variables defined on this space. Consider a non-negative random variable $X$ defined on this probability space and its survival function $S(x)=P(X>x)$. A distortion risk measure applied to $X$, which we denote as $\psi[g: S]$, is defined by

$$
\psi[g: S]=\int_{0}^{+\infty} g(S(x)) d x,
$$

where $g$ is the associated distortion function, which is a function from $[0,1]$ to $[0,1]$ and it must be increasing (not necessarily strictly increasing) and such that $g(0)=0$ and $g(1)=1$. Two main examples of distortion risk measures broadly used in financial and insurance applications are Valueat-Risk (VaR) and Tail Value-at-Risk (TVaR) at a fixed confidence level $\alpha \in(0,1)$, whose distortion functions are $\delta_{\alpha}(t)={ }_{[1-\alpha, 1]}(t)$ and $\gamma_{\alpha}(t)=$ $\left(\frac{t}{1-\alpha}\right) \cdot{ }_{[0,1-\alpha)}(t)+{ }_{[1-\alpha, 1]}(t)$, respectively, where ${ }_{[a, b]}(t)$ equals 1 if $a \leq$ $t \leq b$ and 0 otherwise. Three classes of distortion risk measures that will be used in the rest of the paper and their associated distortion functions are given in Table 1, namely the risk measure based on the Gini's principle, the proportional hazard transform and the dual power transform. Similar procedures can be applied to the Denneberg's absolute deviation principle (Denneberg, 1990), which is defined through the distortion function $g_{\alpha}(t)=$ $t \cdot(1+\alpha) \cdot{ }_{[0,0.5)}(t)+(\alpha+(1-\alpha) \cdot t) \cdot{ }_{[0.5,1]}(t)$ and to the GlueVaR risk measures introduced by Belles-Sampera et al. (2014) which generalize Range$\mathrm{VaR}$ and follow from distortion functions $g_{\beta, \alpha}^{h_{1}, h_{2}}(t)=\left(h_{1} /(1-\beta)\right) \cdot[0,1-\beta)(t)+$ $\left(h_{1}+\left(h_{2}-h_{1}\right) /(\beta-\alpha) \cdot[t-(1-\beta)]\right) \cdot{ }_{[1-\beta, 1-\alpha)}(t)+{ }_{[1-\alpha, 1]}(t)$, with $\alpha \leq$ $\beta<1,0<h_{1}<1, h_{1} \leq h_{2}<1$. Note that when $\alpha=\beta$ neither $(\beta-\alpha)^{-1}$ nor

$[1-\beta, 1-\alpha)(t)$ for $t \in(0,1)$ are well-defined and, in addition, $\alpha=\beta$ implies that $h_{1}=h_{2}$ because $h_{1}$ and $h_{2}$ represent the distorted survival probability associated to $1-\beta$ and $1-\alpha$ respectively. So in those cases the distortion function reduces to $g_{\alpha}^{h_{1}}(t)=\left(h_{1} /(1-\alpha)\right) \cdot{ }_{[0,1-\alpha)}(t)+{ }_{[1-\alpha, 1]}(t)$.

Expression (11) can be understood as the Choquet integral of $X$ with respect to the set function $g \circ P$, where $P$ is the probability function associated with the probability space in which $X$ is defined (Choquet, 1954; Denneberg, 1994). Hereinafter, only non-negative random variables are considered. 
Table 1: Some examples of distortion functions for distortion risk measures

\begin{tabular}{lcl} 
Risk measure & Parameters & Distortion function \\
\hline Gini's principle & $0<\theta<1$ & $g_{\theta}(t)=(1+\theta) \cdot t-\theta \cdot t^{2}$ \\
Proportional Hazard Transform & $m \geq 1$ & $g_{m}(t)=t^{\frac{1}{m}}$ \\
Dual Power Transform & $m \geq 1$ & $g_{m}(t)=1-(1-t)^{m}$ \\
\hline
\end{tabular}
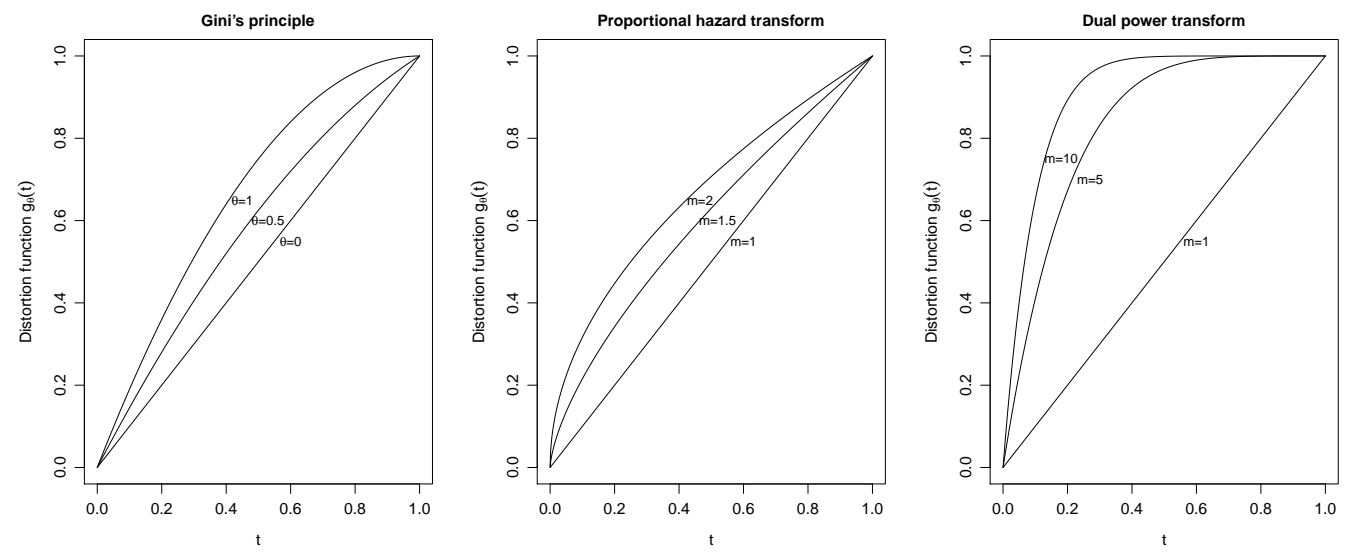

Figure 1: Distortion functions corresponding to Gini's principle (left), proportional hazard transform (center) and dual power transform (right)

The specific preference for a distortion function is difficult to determine. However, the transformation of the survival function reflects in some way the emphasis on the extremes. Belles-Sampera et al. (2016) examined how risk attitudes can be represented in the selection of a given distortion. They showed that the analysis of the distortion function offers a local description of the agents stance on risk in relation to the occurrence of accumulated losses. Here, the concepts of absolute risk attitude and local risk attitude arise naturally. For example, the area under the distortion reveals the global risk attitude, whereas the ratio between the distortion and the identity function provides with local information about the relative risk behavior associated with the risk measure at any point in the range of values

A plot of the three distortion functions (see Figure 1) shows that the Gini's principle risk measure weights the right tail less heavily than the other measures, because its distortion function is flatter than the others for low 
values. When the proportional hazard transform is used, the importance of the large losses is moderate, but when the Dual Power Transform is selected with the parameter equal to 5 or 10 , then we observe a high curve for low values, which means that the right tail of the positive losses will have more importance for the calculation of the risk measure. Therefore, extreme losses are weighted more than in other cases for the Dual Power Transform for $m=10$ because the distortion function is closer to one for low values of $t$.

\section{Distortion risk measures for the non-negative bivariate case}

Let $\left(X_{1}, X_{2}\right)^{\top}$ be a non-negative bivariate random variable with joint survival function $S_{12}\left(x_{1}, x_{2}\right)$ and marginal survival functions $S_{1}\left(x_{1}\right)$ and $S_{2}\left(x_{2}\right)$.

The idea is to introduce distortion risk measures defined on $\left(X_{1}, X_{2}\right)^{\top}$ congruent with unidimensional distortion risk measures defined on the associated marginal distributions.

In a first step, we consider a distortion function $g(\cdot)$, and we define a distorted bivariate survival:

$$
T_{12}\left(x_{1}, x_{2}\right)=g\left[S_{12}\left(x_{1}, x_{2}\right)\right]
$$

where the $g(\cdot)$ function is chosen in order to define a genuine bivariate survival function in (2). Note that the marginal survival functions in (2) are:

$$
T_{1}\left(x_{1}\right)=T_{12}\left(x_{1}, 0\right)=g\left[S_{1}\left(x_{1}\right)\right]
$$

and

$$
T_{2}\left(x_{2}\right)=T_{12}\left(0, x_{2}\right)=g\left[S_{2}\left(x_{2}\right)\right],
$$

which correspond to distorted transformations of the joint survival function $S_{12}\left(x_{1}, x_{2}\right)$.

Once a suitable distortion function $g(\cdot)$ has been selected, a possible distortion risk measure associated to (2) is simply

$$
\psi_{12}\left[g: S_{12}\right]=\int_{0}^{\infty} \int_{0}^{\infty} T_{12}\left(x_{1}, x_{2}\right) d x_{1} d x_{2}=\int_{0}^{\infty} \int_{0}^{\infty} g\left[S_{12}\left(x_{1}, x_{2}\right)\right] d x_{1} d x_{2} .
$$


Note that the corresponding distortion risk measures associated to (3) and (44) are,

$$
\psi_{1}\left[g: S_{1}\right]=\int_{0}^{\infty} g\left[S_{1}\left(x_{1}\right)\right] d x_{1}
$$

and

$$
\psi_{2}\left[g: S_{2}\right]=\int_{0}^{\infty} g\left[S_{2}\left(x_{2}\right)\right] d x_{2} .
$$

So, there is a natural parallelism between the multivariate setting (15) and the marginals in (6) and (7), and the univariate case. This approach is the one proposed in Rüschendorf (2006, section 3) and Rüschendorf (2013, p. 180). However, it is not the only possible way to address risk measures for bivariate risks. See, for instance, Embrechts et al. (2009) that show how multivariate extreme value theory yields the ideal modeling environment. Different extensions to multivariate risk measurement using VaR and TVaR can be found in Cousin and Di Bernardino (2013) and in Cousin and Di Bernardino (2014) who propose vector-valued measures with the same dimension as the underlying risk portfolio, where the lower-orthant risk measure (resp. the upperorthant) is constructed from level sets of multivariate distribution functions (resp. of multivariate survival distribution functions). Contrary to allocation measures or systemic risk measures, these measures are suitable for multivariate risk problems where risks are heterogeneous in nature and cannot be aggregated together before calculating the risk measure.

\section{Some bivariate distortion risk measures with a closed-form expression}

Before generalizing this definition to higher dimensions, we explore some expressions for $\psi_{12}\left[g: S_{12}\right]$ where the $g$ function has been restricted to belong to the set of distortion functions associated to univariate risk measures presented in Table 1. The cases considered here have the advantage of providing a straightforward analytical expression in some cases. The main reason why having closed-form expressions is interesting is because these risk measures can then be implemented in spreadsheet calculations and simulation procedures very easily.

Let us begin with a bivariate random variable $\left(X_{1}, X_{2}\right)^{\top}$ with independent marginals, and then assume a dependence structure between the marginals 
driven by copulas in the Farlie-Gumbel-Morgenstern (FGM) family. In the independence case, we do not assume any particular marginal distribution, but this situation is not the main focus of the article, because what we really want to analyse is cases where we assume a dependence structure. The bivariate Pareto distribution is a clear example of the type of two-dimensional distribution that a risk manager would use to analyse losses coming from two lines of business, or types of risk. For example, in operational risk, we can assume that we have the losses can be of two types and therefore each severity is represented by one of the two dimensions. Similarly, the bivariate exponential distribution or the FGM distribution could reflect the monthly size of losses in internal fraud and external fraud for instance.

A bivariate Pareto distribution for finance/insurance losses is a standard choice. For instance, Embrechts and Puccetti (2006) calculate the bounds of a sum of two Pareto and Log-Normal bivariate risks and they also provide a new definition of multivariate Value-at-Risk.

\subsection{Risk measures for the bivariate independent case}

Let $\left(X_{1}, X_{2}\right)^{\top}$ be a bivariate risk with joint survival function $S_{12}\left(x_{1}, x_{2}\right)$. In this section we obtain bivariate risk measures assuming independence between marginal risks $X_{1}$ and $X_{2}$, that is, assuming that $S_{12}\left(x_{1}, x_{2}\right)=$ $S_{1}\left(x_{1}\right) S_{2}\left(x_{2}\right)$. We consider three different distortion risk measures.

\subsubsection{Risk measures based on the Gini's principle}

Let us consider the distortion function given by the Gini's principle $g_{\theta}(t)=$ $(1+\theta) t-\theta t^{2}$, with $0<\theta<1$. Using (5), we obtain the multivariate measure,

$$
\psi_{12}\left[g_{\theta}: S_{12}\right]=(1+\theta) \cdot \mu_{1} \cdot \mu_{2}-\theta \cdot \mu_{1: 2}^{(1)} \cdot \mu_{1: 2}^{(2)}
$$

where $\mu_{i}=E\left(X_{i}\right), i=1,2$ and $\mu_{1: 2}^{(i)}, i=1,2$ represent the mathematical expectation of the minimum of two copies of the random variable $X_{i}$, with $i=1,2$.

\subsubsection{Risk measures based on the proportional hazard transform}

Let us consider the proportional hazard transform principle given by the distortion function $g_{m}(t)=t^{1 / m}, m \geq 1$. In this case, using notation $F_{i}\left(x_{i}\right)=$ 
$1-S_{i}\left(x_{i}\right), i=1,2$, the multivariate risk measure can be written as

$$
\psi_{12}\left[g_{m}: S_{12}\right]=\prod_{i=1}^{2} E\left\{F_{i}^{-1}[\mathcal{B} e(1,1 / m)]\right\},
$$

where $\mathcal{B} e(a, b)$ represent a classical beta distribution. Note that the terms in the product correspond with the mathematical expectation of the generalized beta distribution (Alexander et al., 2012; Jones et al., 2004, see) with baseline CDF $F_{i}$ and parameters $(1,1 / m)$.

\subsubsection{Risk measures based on the dual power transform}

The following bivariate risk measure is based on the dual power transform principle $g_{m}(t)=1-(1-t)^{m}$ with $m \geq 1$. The corresponding multivariate risk measure is given by

$$
\psi_{12}\left[g_{m}: S_{12}\right]=\sum_{k=1}^{m}(-1)^{k+1}\left(\begin{array}{c}
m \\
k
\end{array}\right) \mu_{1: k}^{(1)} \cdot \mu_{1: k}^{(2)},
$$

where $\mu_{1: k}^{(i)}, i=1,2$ represent the mathematical expectation of the minimum of $k$ copies (iid) of the random variable $X_{i}$, with $i=1,2$. Note that $\mu_{1: 1}^{i}=\mu_{i}$ for all $i$.

\subsection{Risk measures for the bivariate Pareto distribution}

The examples of bivariate risk measures with a closed-form expression which are presented in Section 4.1 are based on the hypothesis of independence between both two risks. However, this assumption is often unrealistic in practice because losses from different sources may occur simultaneously. Then, we work with different classes of dependent risks.

In this section we consider the expressions that several bivariate distortion risk measures take when they are applied to a bivariate dependent Pareto distribution as proposed by Mardia (1962) (see, Arnold (1983)), which sometimes is also called the bivariate Lomax distribution. The bivariate Pareto distribution is defined in terms of the following bivariate survival function

$$
S_{12}\left(x_{1}, x_{2}\right)=\frac{1}{\left(1+x_{1} / \sigma_{1}+x_{2} / \sigma_{2}\right)^{a}}, \quad x_{1}, x_{2} \geq 0,
$$


where $\sigma_{1}, \sigma_{2}>0$ are scale parameters and $a>0$ is a shape parameter. Note that both marginal distributions are Pareto distributions with survival functions equal to $S_{i}\left(x_{i}\right)=\frac{1}{\left(1+x_{i} / \sigma_{i}\right)^{a}}$, with $x_{i} \geq 0, i=1,2$.

In order to compute the different bivariate risk measures we use the result stated in Lemma 1.

Lemma 1 If $\sigma_{1}, \sigma_{2}>0$ and $a>2$, then

$$
\int_{0}^{\infty} \int_{0}^{\infty} \frac{d x_{1} d x_{2}}{\left(1+x_{1} / \sigma_{1}+x_{2} / \sigma_{2}\right)^{a}}=\frac{\sigma_{1} \sigma_{2}}{(a-1)(a-2)} .
$$

Proof: The result is direct taking into account that if $a>1$,

$$
\int_{0}^{\infty} \frac{d x_{1}}{\left(1+x_{1} / \sigma_{1}+x_{2} / \sigma_{2}\right)^{a}}=\frac{\sigma_{1}}{a-1} \frac{1}{\left(1+x_{2} / \sigma_{2}\right)^{a-1}} .
$$

\subsubsection{Risk measures based on the Gini's principle}

If we consider the distortion function given by the Gini's principle $g_{\theta}(t)=$ $(1+\theta) t-\theta t^{2}$, with $0<\theta<1$, using (5) and (12) we obtain

$$
\psi_{12}\left[g_{\theta}: S_{12}\right]=(1+\theta) \frac{\sigma_{1} \sigma_{2}}{(a-1)(a-2)}-\theta \frac{\sigma_{1} \sigma_{2}}{(2 a-1)(2 a-2)},
$$

which can be written as

$$
\psi_{12}\left[g_{\theta}: S_{12}\right]=\frac{(3 a \theta+4 a-2) \sigma_{1} \sigma_{2}}{2(a-1)(a-2)(2 a-1)},
$$

and is valid for $a>2$.

\subsubsection{Risk measures based on the proportional hazard transform}

Now, we choose the proportional hazard transform principle represented by the distortion function $g_{m}(t)=t^{1 / m}$ with $m \geq 1$. The associated risk measure is

$$
\psi_{12}\left[g_{m}: S_{12}\right]=\frac{m \sigma_{1} \sigma_{2}}{(a-m)(a-2 m)}
$$

if $a>2 m$. 


\subsubsection{Risk measures based on the dual power transform}

For the dual power transform principle with distortion function $g_{m}(t)=$ $1-(1-t)^{m}$ with $m \geq 1$, the corresponding bivariate risk measure is given by

$$
\psi_{12}\left[g_{m}: S_{12}\right]=\sum_{k=1}^{m}(-1)^{k+1}\left(\begin{array}{c}
m \\
k
\end{array}\right) \frac{\sigma_{1} \sigma_{2}}{(a k-1)(a k-2)},
$$

with $a>2$.

\subsection{Risk measures for the bivariate exponential distri- bution}

Another dependence structure to be investigated is the bivariate exponential distribution given by

$$
S_{12}\left(x_{1}, x_{2}\right)=\exp \left(-a_{1} x_{1}-a_{2} x_{2}-\phi a_{1} a_{2} x_{1} x_{2}\right), \quad x_{1}, x_{2} \geq 0,
$$

where $a_{1}, a_{2}>0$ and $0 \leq \phi \leq 1$. This joint survival function corresponds to the Gumbel type I bivariate exponential distribution considered by Gumbel (1960).

The following lemma is useful for the computation of the different risk measures when they are applied to this distribution.

Lemma 2 If $S_{12}\left(x_{1}, x_{2}\right)$ denotes the bivariate survival function defined in (16), we have

$$
\int_{0}^{\infty} \int_{0}^{\infty} S_{12}\left(x_{1}, x_{2}\right) d x_{1} d x_{2}=\frac{\exp (1 / \phi)}{\phi a_{1} a_{2}}[-E i(1 / \phi)]
$$

where

$$
-E i(z)=\int_{z}^{\infty} \frac{e^{-t}}{t} d t
$$

represents the exponential integral function.

Proof: Integrating (16) with respect $x_{1}$ we have,

$$
\int_{0}^{\infty} S_{12}\left(x_{1}, x_{2}\right) d x_{1}=\frac{e^{-a_{2} x_{2}}}{a_{1}\left(1+a_{2} \phi x_{2}\right)},
$$

and integrating again with respect $x_{2}$ we obtain (17), using definition (18). 


\subsubsection{Risk measures based on the Gini's principle}

For the Gini's principle we have that the risk measure expression for the bivariate exponential distribution is

$$
\psi_{12}\left[g_{\theta}: S_{12}\right]=\frac{(1+\theta) \exp (1 / \phi)}{\phi a_{1} a_{2}}[-E i(1 / \phi)]-\frac{\theta \exp (2 / \phi)}{2 \phi a_{1} a_{2}}[-E i(2 / \phi)] .
$$

\subsubsection{Risk measures based on the proportional hazard transform}

In the case of the proportional hazard transform we obtain that the bivariate risk measure can be expressed as

$$
\psi_{12}\left[g_{m}: S_{12}\right]=\frac{m \exp (1 / m \phi)}{\phi a_{1} a_{2}}[-E i(1 / m \phi)]
$$

\subsubsection{Risk measures based on the dual power transform principle}

For the dual power transform principle we obtain the following closed-form expression for the risk measure applied to a bivariate exponential distribution:

$$
\psi_{12}\left[g_{m}: S_{12}\right]=\sum_{k=1}^{m}(-1)^{k+1}\left(\begin{array}{c}
m \\
k
\end{array}\right) \frac{\exp (k / \phi)}{k \phi a_{1} a_{2}}[-E i(k / \phi)] .
$$

\subsection{A dependent model based on the FGM distribu- tions}

Now, we consider the Farlie-Gumbel-Morgenstern distribution (Farlie, 1960; Gumbel, 1960; Morgenstern, 1956) with joint survival function,

$$
S_{12}\left(x_{1}, x_{2} ; \alpha\right)=S_{1}\left(x_{1}\right) S_{2}\left(x_{2}\right)\left[1+\delta\left(1-S_{1}\left(x_{1}\right)\right)\left(1-S_{2}\left(x_{2}\right)\right)\right]
$$

where $\delta \in[-1,1]$ is the dependence parameter, and $\delta=0$ corresponds to the independent case.

For obtaining the different bivariate risk measures, we need the following lemma.

Lemma 3 Let $X_{i: n}$ be the ith order statistics in a sample of size $n$ and let the ith spacing be

$$
S_{i: n}=X_{i+1: n}-X_{i: n}
$$


The fundamental formulae for moments of order statistics in terms of integrals concerning distribution function only is given by,

$$
E\left(S_{i: n}\right)=\left(\begin{array}{c}
n \\
i
\end{array}\right) \int_{-\infty}^{\infty} F(x)^{i}[1-F(x)]^{n-i} d x .
$$

Proof: See Pearson (1902) and also Jones and Balakrishnan (2002).

Using Lemma 3, if $X$ is a positive random variable we have,

$$
\begin{aligned}
\int_{0}^{\infty} F(x)[1-F(x)] d x & =\frac{E\left(S_{1: 2}\right)}{2}=\frac{E\left(X_{2: 2}-X_{1: 2}\right)}{2}, \\
\int_{0}^{\infty} F(x)[1-F(x)]^{2} d x & =\frac{E\left(S_{1: 3}\right)}{3}=\frac{E\left(X_{2: 3}-X_{1: 3}\right)}{3}, \\
\int_{0}^{\infty} F(x)^{2}[1-F(x)]^{2} d x & =\frac{E\left(S_{2: 4}\right)}{6}=\frac{E\left(X_{3: 4}-X_{2: 4}\right)}{6} .
\end{aligned}
$$

\subsubsection{Risk measures based on the Gini's principle}

We consider the distortion function based on the Gini's principle given by $g_{\theta}(t)=(1+\theta) t-\theta t^{2}$. We have the following theorem.

Theorem 1 Let $\left(X_{1}, X_{2}\right)^{\top}$ a bivariate random variable with bivariate survival function given by (22). Then, we have

$$
\int_{0}^{\infty} \int_{0}^{\infty} S_{12}\left(x_{1}, x_{2}\right) d x_{1} d x_{2}=\mu_{1} \mu_{2}+\frac{\delta}{4} E\left(S_{1: 2}^{(1)}\right) E\left(S_{1: 2}^{(2)}\right)
$$

and

$\int_{0}^{\infty} \int_{0}^{\infty} S_{12}\left(x_{1}, x_{2}\right)^{2} d x_{1} d x_{2}=\mu_{1: 2}^{(1)} \mu_{1: 2}^{(2)}+\frac{2 \delta}{9} E\left(S_{1: 3}^{(1)}\right) E\left(S_{1: 3}^{(2)}\right)+\frac{\delta^{2}}{36} E\left(S_{2: 4}^{(1)}\right) E\left(S_{2: 4}^{(2)}\right)$,

where $S_{i: n}^{(k)}, k=1,2$ is defined in (23), where the superscript corresponds to the marginal $X_{k}, k=1,2$ and $\mu_{1: 2}^{i}, i=1,2$ are defined as in section 4.1.1.

Proof: The proof is direct using the expression of the survival FGM copula (22) and formulas (25) to (27).

Using the previous result, the corresponding bivariate risk measure is:

$$
\begin{aligned}
\psi_{12}\left[g_{\theta}: S_{12}\right] & =(1+\theta)\left\{\mu_{1} \mu_{2}+\frac{\delta}{4} E\left(S_{1: 2}^{(1)}\right) E\left(S_{1: 2}^{(2)}\right)\right\}- \\
& -\theta\left\{\mu_{1: 2}^{(1)} \mu_{1: 2}^{(2)}+\frac{2 \delta}{9} E\left(S_{1: 3}^{(1)}\right) E\left(S_{1: 3}^{(2)}\right)+\frac{\delta^{2}}{36} E\left(S_{2: 4}^{(1)}\right) E\left(S_{2: 4}^{(2)}\right)\right\}
\end{aligned}
$$




\subsubsection{Risk measures based on the dual power transform}

If we take the distortion function $g_{m}(t)=1-(1-t)^{m}$ with $m=2$ we obtain the formula,

$$
\begin{aligned}
\psi_{12}\left[g_{2}: S_{12}: \delta\right] & =2 \mu_{1} \cdot \mu_{2}-\mu_{1: 2}^{(1)} \cdot \mu_{1: 2}^{(2)} \\
& +\frac{2 \delta}{4}\left(\mu_{2: 2}^{(1)}-\mu_{1: 2}^{(1)}\right) \cdot\left(\mu_{2: 2}^{(2)}-\mu_{1: 2}^{(2)}\right) \\
& -\frac{2 \delta}{9}\left(\mu_{2: 3}^{(1)}-\mu_{1: 3}^{(1)}\right) \cdot\left(\mu_{2: 3}^{(2)}-\mu_{1: 3}^{(2)}\right) \\
& -\frac{\delta^{2}}{36}\left(\mu_{3: 4}^{(1)}-\mu_{2: 4}^{(1)}\right) \cdot\left(\mu_{3: 4}^{(2)}-\mu_{2: 4}^{(2)}\right)
\end{aligned}
$$

where,

$$
\mu_{i: j}^{(k)}=E\left[X_{i: j}^{(k)}\right], \quad k=1,2,
$$

and $X_{i: j}^{(k)}, k=1,2$ is the $i$ th-order statistics in a sample of size $j$ corresponding to the random variables $X_{1}$ and $X_{2}$.

If we set $\delta=0$ in (31) we obtain (10), taking into account that $\mu_{1: 1}(i)=\mu_{i}$ for all $i=1,2$.

\section{Extension to the multivariate case}

Let us consider $\left(X_{1}, X_{2}, \ldots, X_{p}\right)^{\top}$ a $p$-dimensional non-negative random variable and a distortion function $g$. Following the spirit of the definition of distortion risk measures for the non-negative bivariate case given in expression (5), the distortion risk measure for multivariate risks associated to $g$ may be defined by

Definition 1 A distortion risk measure for multivariate non-negative risks can be defined as:

$$
\psi_{12 \ldots p}\left[g: S_{12 \ldots p}\right]=\int_{0}^{\infty} \cdots \int_{0}^{\infty} g\left[S_{12 \ldots p}\left(x_{1}, \ldots, x_{p}\right)\right] d x_{1} \cdots d x_{p},
$$

where $S_{12 \ldots p}$ is the multivariate survival function of $p$-dimensional non-negative random variable $\left(X_{1}, X_{2}, \ldots, X_{p}\right)^{\top}$. and $g$ is a distortion function.

This definition corresponds to the one given in section 3 of Rüschendorf (2006). 
Definition 1 may not be too appropriate for some specific purposes. For instance, if an insurance company needs to determine a solvency capital for a three-year window, it is necessary that the risk value preserves the scale, so it should correspond to monetary units and not, for instance, to "monetary units to the power of three'. If $X_{s}$ is the random loss from period $s-1$ to period $s, s=1,2,3$, then an insurance company interested in a risk measure for vector $\left(X_{1}, X_{2}, X_{3}\right)^{\top}$ may find that $\psi_{123}\left[g: S_{123}\right]$ is too large. Our proposal is to consider $\left(\psi_{123}\left[g: S_{123}\right]\right)^{1 / 3}$ to overcome such an inconvenience.

Definition 2 A rescaled distortion risk measure for multivariate non-negative risks can be defined as:

$$
\rho_{12 \ldots p}\left[g: S_{12 \ldots p}\right]=\left(\psi_{12 \ldots p}\left[g: S_{12 \ldots p}\right]\right)^{1 / p},
$$

where $\psi_{12 \ldots p}\left[g: S_{12 \ldots p}\right]$ comes from definition $1, S_{12 \ldots p}$ is the multivariate survival function of $p$-dimensional non-negative random variable $\left(X_{1}, X_{2}, \ldots, X_{p}\right)^{\top}$. and $g$ is a distortion function.

It has to be noted that, once a distortion function $g$ has been selected, both definitions 1 and 2 are consistent with the definition of a distortion risk measure for the unidimensional case, because $\rho_{1}\left[g: S_{1}\right]=\psi_{1}\left[g: S_{1}\right]$ by expression (33) and they also match expression (11).

We suggest that standardised data could be used when the different units of measurement are a concern. In many cases the dimensions use different units of measurement. For instance, in the industry of financial services, some risks are price-based (such as the betas), whereas others are calculated as an index (Composite Indicator of Systemic Stress) or balance-sheet based (the ratio of nonperforming loans over total loans).

In this section we only compute the multivariate risk measure (32), assuming that the components of the random vector $\left(X_{1}, \ldots, X_{p}\right)^{\top}$ are independent, because in this case the expressions are straightforward.

For the distortion function given by the Gini's principle $g_{\theta}(t)=(1+\theta) t-$ $\theta t^{2}$, with $0<\theta<1$, expression (32) turns into

$$
\psi_{12 \ldots p}\left[g_{\theta}: S_{12 \ldots p}\right]=(1+\theta) \prod_{i=1}^{p} \mu_{i}-\theta \prod_{i=1}^{p} \mu_{1: 2}^{(i)},
$$

where $\mu_{i}=E\left(X_{i}\right), i=1,2$ and $\mu_{1: 2}^{(i)}, i=1,2, \ldots, p$ represent the mathematical expectation of the minimum of two copies of the random variable $X_{i}$, with $i=1,2$. 
In this case, the closed-form expression of the multivariate risk measure for independent risks is

$$
\psi_{12 \ldots p}\left[g_{m}: S_{12 \ldots p}\right]=\prod_{i=1}^{p} E\left\{F_{i}^{-1}[\mathcal{B} e(1,1 / m)]\right\}
$$

where $\mathcal{B} e(a, b)$ represent a classical beta distribution.

For the dual power transform principle $g_{m}(t)=1-(1-t)^{m}$ with $m \geq 1$, the expression for the multivariate risk measure is given by,

$$
\psi_{12 \ldots p}\left[g_{m}: S_{12 \ldots p}\right]=\sum_{k=1}^{m}(-1)^{k+1}\left(\begin{array}{c}
m \\
k
\end{array}\right) \prod_{i=1}^{p} \mu_{1: k}^{(i)},
$$

where $\mu_{1: k}^{(i)}, i=1,2, \ldots, p$ represent the mathematical expectation of the minimum of $k$ copies (iid) of the random variable $X_{i}$, with $i=1,2, \ldots, p$.

\section{Numerical example for bivariate non-negative risks}

We considered an example where the occurrence of two phenomena is observed over time. The objective is to provide a multivariate risk measure in order to monitor the evolution of risk of those two magnitudes using one single synthesized value. Therefore, one multivariate risk measure is better than using two different risk measures for each dimension separately.

This application shows that it is possible to analyze multivariate operational risk from many sources, for instance when the risk manager has to monitor the occurrence of operational events by looking at the number or severity of events by classes, i.e. several dimensions, and he wants to have only one risk value instead of a different risk measure for every type of event.

\subsection{Data and methodology}

For illustrative purposes, we took accident (unintentional injury) deaths data from the Spanish National Statistics Institute ((INE, 2014)). In that dataset, accident deaths in Spain are classified according to the following list of causes: (1) traffic accidents of motor vehicles; (2) other transport accidents; (3) accidental falls; (4) accidental drowning, immersion and suffocation; (5) accidents 
by fire, smoke and hot substances; (6) accidental poisoning by psychoactive drugs and abuse drugs; (7) other accidental poisoning; (8) other accidents. For this work, we grouped them into two classes: deaths due to crashes (causes (1)-(2)) and deaths due to other accidental causes (causes (3)-(8)). Then, we analyzed the following bivariate variable: number of fatalities due to crashes $(X 1)$ and number of deaths due to other accidental causes $(X 2)$, in a province or autonomous city (according to the province of residence of the deceased), in a year - there are 50 provinces and 2 autonomous cities in Spain. For that, we selected the following four years: 2000, 2004, 2008 and 2012. Table 2 shows the dataset considered and Figure 2 shows the corresponding three-dimensional histograms.
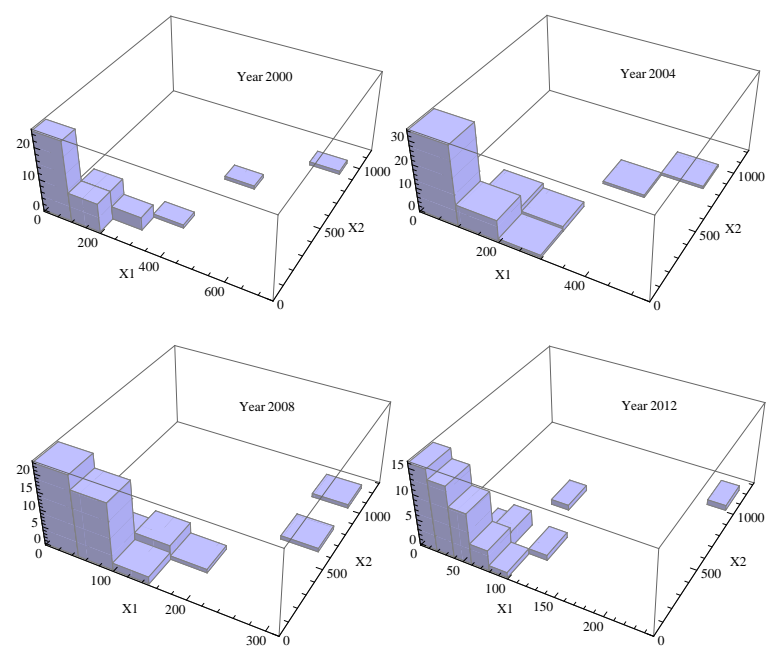

Figure 2: Display of 3-dimensional histograms: fatalities due to crashes $(X 1)$ and deaths due to other accidental causes $(X 2)$, in a Spanish province, in a year. Years: 2000, 2004, 2008, 2012.

Given that the observed number of occurences is large, we did not fit a discrete distribution, but we fitted the bivariate Pareto distribution described in Section 4.1 by maximum likelihood. For that model, the probability density function is

$$
f_{12}\left(x_{1}, x_{2} ; a, \sigma_{1}, \sigma_{2}\right)=\frac{\partial^{2} S_{12}\left(x_{1}, x_{2}\right)}{\partial x_{1} \partial x_{2}}=\frac{a(a+1)}{\sigma_{1} \sigma_{2}\left(1+\frac{x_{1}}{\sigma_{1}}+\frac{x_{2}}{\sigma_{2}}\right)^{a+2}},
$$


Table 2: Accident deaths in Spain, due to crashes $(X 1)$ or other accidental causes $(X 2)$, in a province (or autonomous city). Years: 2000, 2004, 2008 and 2012. Source: INE.

\begin{tabular}{|c|c|c|c|c|c|c|c|c|}
\hline \multirow{2}{*}{ Province $\backslash$ Year } & \multicolumn{2}{|c|}{2000} & \multicolumn{2}{|c|}{2004} & \multicolumn{2}{|c|}{2008} & \multicolumn{2}{|c|}{2012} \\
\hline & $X 1$ & $X 2$ & $X 1$ & $X 2$ & $X 1$ & $X 2$ & $X 1$ & $X 2$ \\
\hline Albacete & 64 & 44 & 44 & 48 & 30 & 69 & 17 & 94 \\
\hline Alicante/Alacant & 219 & 165 & 198 & 209 & 133 & 255 & 65 & 226 \\
\hline Almería & 129 & 75 & 114 & 81 & 56 & 96 & 31 & 103 \\
\hline Araba/Alava & 43 & 27 & 37 & 39 & 19 & 43 & 16 & 53 \\
\hline Asturias & 188 & 181 & 151 & 245 & 74 & 241 & 66 & 297 \\
\hline Avila & 19 & 17 & 27 & 37 & 11 & 32 & 15 & 45 \\
\hline Badajoz & 107 & 57 & 100 & 66 & 64 & 54 & 40 & 99 \\
\hline Balears, Illes & 147 & 124 & 118 & 118 & 93 & 137 & 67 & 164 \\
\hline Barcelona & 646 & 902 & 414 & 940 & 253 & 1192 & 226 & 1101 \\
\hline Bizkaia & 168 & 148 & 106 & 168 & 61 & 195 & 51 & 200 \\
\hline Burgos & 92 & 42 & 42 & 87 & 44 & 79 & 31 & 85 \\
\hline Cáceres & 54 & 45 & 80 & 54 & 33 & 32 & 16 & 58 \\
\hline Cádiz & 123 & 87 & 129 & 131 & 61 & 133 & 38 & 140 \\
\hline Cantabria & 69 & 83 & 43 & 101 & 31 & 120 & 24 & 178 \\
\hline Castellón/Castelló & 100 & 68 & 87 & 75 & 41 & 74 & 31 & 89 \\
\hline Ciudad Real & 88 & 47 & 60 & 63 & 56 & 73 & 31 & 114 \\
\hline Córdoba & 91 & 84 & 82 & 98 & 57 & 112 & 47 & 119 \\
\hline Coruña, A & 251 & 160 & 169 & 211 & 105 & 229 & 65 & 163 \\
\hline Cuenca & 33 & 26 & 36 & 32 & 18 & 49 & 18 & 65 \\
\hline Gipuzkoa & 110 & 109 & 67 & 113 & 49 & 120 & 22 & 136 \\
\hline Girona & 94 & 106 & 71 & 119 & 51 & 130 & 48 & 132 \\
\hline Granada & 119 & 115 & 112 & 125 & 80 & 134 & 49 & 122 \\
\hline Guadalajara & 25 & 18 & 28 & 29 & 15 & 31 & 12 & 44 \\
\hline Huelva & 46 & 42 & 51 & 54 & 44 & 44 & 25 & 62 \\
\hline Huesca & 47 & 27 & 43 & 50 & 19 & 44 & 19 & 49 \\
\hline Jaén & 84 & 72 & 68 & 98 & 75 & 66 & 20 & 80 \\
\hline León & 121 & 72 & 78 & 96 & 69 & 99 & 48 & 121 \\
\hline Lleida & 100 & 61 & 86 & 76 & 56 & 94 & 43 & 75 \\
\hline Lugo & 100 & 73 & 79 & 76 & 50 & 78 & 37 & 85 \\
\hline Madrid & 488 & 665 & 357 & 782 & 253 & 767 & 86 & 696 \\
\hline Málaga & 150 & 135 & 142 & 170 & 113 & 189 & 63 & 240 \\
\hline Murcia & 222 & 132 & 218 & 174 & 118 & 178 & 80 & 172 \\
\hline Navarra & 108 & 68 & 94 & 106 & 52 & 115 & 40 & 114 \\
\hline Ourense & 76 & 69 & 64 & 117 & 40 & 90 & 21 & 94 \\
\hline Palencia & 34 & 19 & 24 & 37 & 17 & 38 & 6 & 44 \\
\hline Palmas, Las & 132 & 142 & 63 & 192 & 111 & 204 & 31 & 101 \\
\hline Pontevedra & 186 & 133 & 136 & 211 & 94 & 170 & 70 & 157 \\
\hline Rioja, La & 60 & 42 & 55 & 62 & 36 & 53 & 14 & 49 \\
\hline Salamanca & 44 & 34 & 61 & 58 & 36 & 64 & 19 & 51 \\
\hline Santa Cruz de Tenerife & 89 & 109 & 73 & 147 & 59 & 164 & 48 & 170 \\
\hline Segovia & 32 & 15 & 21 & 17 & 11 & 26 & 10 & 18 \\
\hline Sevilla & 204 & 180 & 216 & 213 & 121 & 213 & 72 & 196 \\
\hline Soria & 24 & 13 & 32 & 17 & 12 & 34 & 3 & 17 \\
\hline Tarragona & 143 & 116 & 130 & 120 & 72 & 157 & 48 & 137 \\
\hline Teruel & 33 & 22 & 23 & 38 & 16 & 40 & 10 & 21 \\
\hline Toledo & 81 & 55 & 79 & 77 & 66 & 74 & 32 & 147 \\
\hline Valencia/València & 311 & 274 & 286 & 300 & 183 & 307 & 101 & 360 \\
\hline Valladolid & 70 & 57 & 66 & 87 & 44 & 71 & 23 & 72 \\
\hline Zamora & 37 & 31 & 28 & 35 & 17 & 31 & 7 & 35 \\
\hline Zaragoza & 150 & 86 & 145 & 114 & 85 & 150 & 54 & 117 \\
\hline Ceuta & 4 & 4 & 9 & 4 & 2 & 6 & 2 & 9 \\
\hline Melilla & 4 & 5 & 3 & 9 & 2 & 8 & 0 & 7 \\
\hline
\end{tabular}


where $\left(a, \sigma_{1}, \sigma_{2}\right)$ is the unknown three-parameter vector of the model, $S_{12}\left(x_{1}, x_{2}\right)$ is the corresponding bivariate survival function (Eq. 11), and the log-likelihood function is given by

$$
\begin{aligned}
& \log \ell\left(a, \sigma_{1}, \sigma_{2}\right)=\sum_{i=1}^{n} \log f\left(x_{1 i}, x_{2 i} ; a, \sigma_{1}, \sigma_{2}\right)= \\
& \quad=n \log [a(a+1)]-n \log \left(\sigma_{1}\right)-n \log \left(\sigma_{2}\right)-(a+2) \sum_{i=1}^{n} \log \left(1+\frac{x_{1 i}}{\sigma_{1}}+\frac{x_{2 i}}{\sigma_{2}}\right)
\end{aligned}
$$

where $\left(x_{1 i}, x_{2 i}\right), i=1, \ldots, n$ is the sample bivariate data and the maximum likelihood estimation of the parameter vector $\left(\hat{a}, \hat{\sigma}_{1}, \hat{\sigma}_{2}\right)$ is the one that maximizes the log-likelihood function $\log \ell\left(a, \sigma_{1}, \sigma_{2}\right)$.

Finally, we obtained the risk measures for the bivariate Pareto distribution based on the Gini's principle, on the proportional hazard trans-

form and on the dual power transform, described in Section 4.1. by using Eqs. (13),14, 15).

\subsection{Results}

Table 3 shows the parameter estimates from the bivariate Pareto model considered ( $a, \sigma_{1}$ and $\sigma_{2}$ parameters), fitted to the number of fatalities due to crashes and number of deaths due to other accidental causes, in a Spanish province or autonomous city, in a year, by maximum likelihood and in the four years selected: 2000, 2004, 2008 and 2012.

Tables 4, 5 and 6] show the risk measures for the bivariate Pareto distribution based on the Gini's principle, based on the proportional hazard transform and based on the dual power transform, respectively.

It can be seen that increasing the value of $\theta$ (Table 4) or the value of $m$ (Tables 5, 6) results in an increase in the corresponding risk measure value. Additionally, in this example, it can be seen that risk measures decrease in most cases year-over-year when $\theta$ or $m$ are held constant.

The conclusion for this illustration is that there is evidence of a descent in the risk of the number of deaths by two different causes from 2000 to 2012.

This application shows that our proposed method to quantify multivariate operational risk is a straightforward method that is useful to monitor multivariate risks. 
Table 3: Parameter estimates from the bivariate Pareto model to the accident deaths dataset by maximum likelihood. Years: 2000, 2004, 2008 and 2012.

\begin{tabular}{ccccc}
\hline & 2000 & 2004 & 2008 & 2012 \\
\hline$\hat{a}$ & 4.2406 & 5.7511 & 5.1390 & 6.1394 \\
$\hat{\sigma}_{1}$ & 395.43 & 471.63 & 271.84 & 206.63 \\
$\hat{\sigma}_{2}$ & 321.96 & 590.56 & 544.90 & 699.24 \\
\hline
\end{tabular}

When looking at the plots of the distortion functions presented in Section 2 , we clearly see that for all of them, the larger the parameter, the closer is the distortion function to one for low values of $t$. In the distortion, low values of $t$ correspond exactly to large values of the loss variables. Therefore we expect to obtain risk measures that increase when the distortion parameter increases. In Tables 4, 5 and 6 , we see that the larger the value of $\theta$ and $m$, then the larger is the resulting risk value. This happens for all the years (columns) and all the risk measures. The reason is that the larger this parameter, then the larger is the weight of the right tail of the loss distribution in the computation of the risk summary value.

When we look at the risk values by rows, we always obtain a descending trend. This would not happen if we were using a concave transform that would not weight the large value of losses so much.

In Figure 3, we have decided to plot the trend over the years of risk values based on $\theta$ equal to 0.5 for the Ginis principle, then $m$ equal to 1.5 for the proportional hazard transform and $m$ equal to 5 for the dual power transform. They correspond to the mid row of Tables 4, 5 and 6 , respectively. These values are powerful indicators that are able to capture the multivariate structure of risks and to represent that in one single value per year. When looking at the trend presented in Figure 3, we conclude that there has been a clear decreasing risk over the years, when taking into consideration the two dimensions of losses.

We have shown that the multivariate risk measure analysis provides a simple tool to monitor the evolution of risk over the years when we take into account the two dimensions considered in this example, which were the number of victims by type. We liked this particular example because it is common to have several types of operational risk events which need to be monitored over time and simultaneously. 
Table 4: Risk measures for the bivariate Pareto distribution based on the Gini's principle for accident deaths bivariate data. Years: 2000, 2004, 2008 and 2012.

\begin{tabular}{ccccc}
\hline$\theta$ & 2000 & 2004 & 2008 & 2012 \\
\hline 0.0 & 17534.7 & 15628.0 & 11400.8 & 6791.6 \\
0.1 & 19025.6 & 16911.7 & 12348.0 & 7346.2 \\
0.2 & 20516.5 & 18195.4 & 13295.2 & 7900.7 \\
0.3 & 22007.4 & 19479.1 & 14242.4 & 8455.3 \\
0.4 & 23498.3 & 20762.8 & 15189.7 & 9009.8 \\
0.5 & 24989.2 & 22046.5 & 16136.9 & 9564.3 \\
0.6 & 26480.1 & 23330.2 & 17084.1 & 10118.9 \\
0.7 & 27971.0 & 24613.9 & 18031.3 & 10673.4 \\
0.8 & 29461.8 & 25897.6 & 18978.5 & 11227.9 \\
0.9 & 30952.7 & 27181.3 & 19925.8 & 11782.5 \\
1.0 & 32443.6 & 28465.0 & 20873.0 & 12337.0 \\
\hline
\end{tabular}

Table 5: Risk measures for the bivariate Pareto distribution based on the proportional hazard transform for accident deaths bivariate data. Years: 2000, 2004, 2008 and 2012.

\begin{tabular}{ccccc}
\hline$m$ & 2000 & 2004 & 2008 & 2012 \\
\hline 1.0 & 17534.7 & 15628.0 & 11400.8 & 6791.6 \\
1.1 & 21853.0 & 18549.4 & 13725.9 & 8005.9 \\
1.2 & 27299.4 & 21914.5 & 16474.9 & 9387.1 \\
1.3 & 34308.1 & 25814.7 & 19755.2 & 10966.0 \\
1.4 & 43558.0 & 30366.8 & 23711.6 & 12780.9 \\
1.5 & 56170.7 & 35722.1 & 28544.1 & 14880.2 \\
1.6 & 74136.6 & 42080.6 & 34536.5 & 17325.6 \\
1.7 & 101351.0 & 49711.5 & 42104.9 & 20197.3 \\
1.8 & 146589.0 & 58985.5 & 51883.7 & 23601.3 \\
1.9 & 234589.0 & 70427.1 & 64889.3 & 27680.2 \\
2.0 & 472423.0 & 84802.8 & 82855.8 & 32630.6 \\
\hline
\end{tabular}

Table 6: Risk measures for the bivariate Pareto distribution based on the dual power tranform for accident deaths bivariate data. Years: 2000, 2004, 2008 and 2012.

\begin{tabular}{ccccc}
\hline$m$ & 2000 & 2004 & 2008 & 2012 \\
\hline 1 & 17534.7 & 15628.0 & 11400.8 & 6791.6 \\
2 & 32443.6 & 28465.0 & 20873.0 & 12337.0 \\
3 & 45739.8 & 39634.5 & 29182.3 & 17141.3 \\
4 & 57903.2 & 49657.3 & 36686.4 & 21437.9 \\
5 & 69208.8 & 58826.7 & 43587.7 & 25357.8 \\
6 & 79832.9 & 67327.8 & 50014.7 & 28983.4 \\
7 & 89896.8 & 75286.5 & 56055.1 & 32370.7 \\
8 & 99488.7 & 82793.2 & 61772.2 & 35559.8 \\
9 & 108675.0 & 89915.4 & 67213.4 & 38580.6 \\
10 & 117508.0 & 96705.4 & 72415.4 & 41456.1 \\
\hline
\end{tabular}



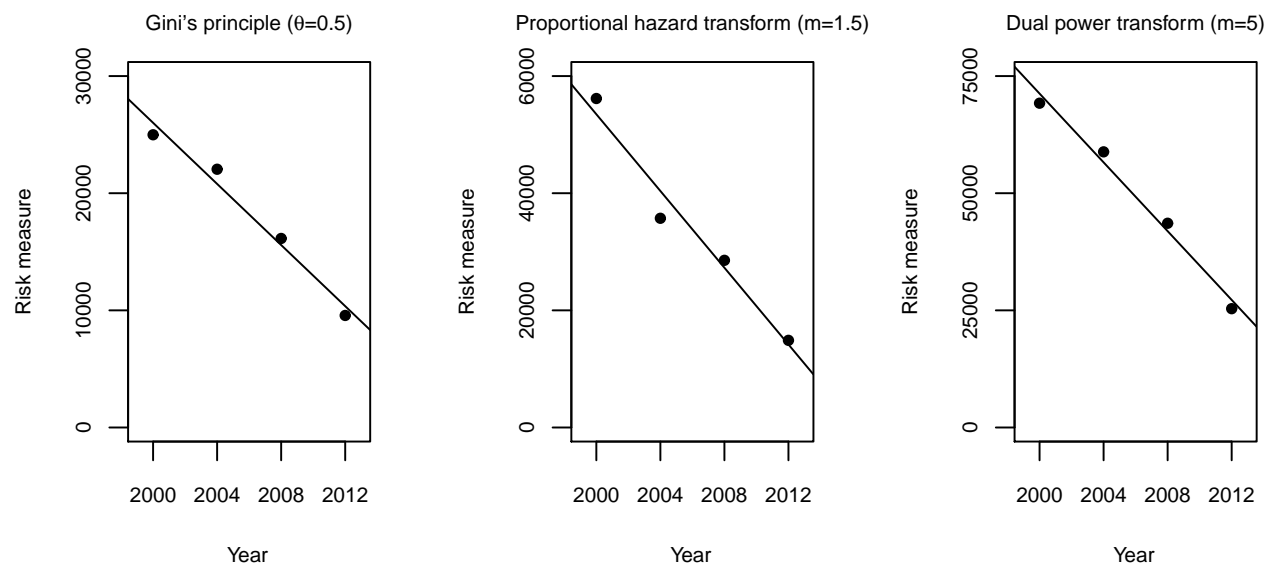

Figure 3: Trend over the years of risk values based on $\theta=0.5$ for the Gini's principle (left), $m=1.5$ for the proportional hazard transform (center), $m=5$ for the dual power transform (right). Years: 2000, 2004, 2008 and 2012 .

\section{Conclusions}

We have presented a way to address the definition of multivariate distortion risk measures and we have given some examples of distortion functions and distributions where the final expression has a closed-form.

We believe that this methodological approach, although it is restricted to non-negative cases, can be useful in many risk management applications.

The main advantage of our method is that there is no need to use vectorvalue risk measures and that for some distributions which are typical in the operational risk context such as the bivariate Pareto, we can obtain analytic expressions for the multivariate distortion risk measures. The main drawback is the interpretation of the summarizing measure in the scale and units of the original components of the vector of losses.

The main limitation regarding interpretation, like in many other aggregation methods, is that distortion functions combine and rescale the original units of measurement. In the multivariate case, when we use distorted multivariate survival functions to obtain a distortion risk measure for a multivariate risk, the units of measurement are also distorted. 


\section{Acknowledgements}

The support received from the Spanish Ministry of Science/FEDER ECO201676203-C2-1-P and ECO2016-76203-C2-2-P is acknowledged.

\section{Declarations of Interest}

The authors report no conflicts of interest. The authors alone are responsible for the content and writing of the paper.

\section{References}

C. Alexander, G.M. Cordeiro, E.M.M. Ortega, and J.M. Sarabia. Generalized beta-generated distributions. Computational Statistics and Data Analysis, 56(6):1880-1897, 2012. doi: 10.1016/j.csda.2011.11.015.

B.C. Arnold. Pareto Distributions. International Co-operative Publishing House, Fairland, MD, 1983. ISBN 089974012X.

J. Belles-Sampera, J.M. Merigó, M. Guillen, and M. Santolino. The connection between distortion risk measures and ordered weighted averaging operators. Insurance: Mathematics and Economics, 52(2):411-420, 2013. doi: 10.1016/j.insmatheco.2013.02.008.

J. Belles-Sampera, M. Guillen, and M. Santolino. Beyond value-at-risk: Gluevar distortion risk measures. Risk Analysis, 34(1):121-134, 2014. doi: $10.1111 /$ risa. 12080 .

J. Belles-Sampera, M. Guillen, and M. Santolino. What attitudes to risk underlie distortion risk measure choices? Insurance: Mathematics and Economics, 68:101-109, 2016. doi: 10.1016/j.insmatheco.2016.02.005.

Gustave Choquet. Theory of capacities. Annales de l'Institut Fourier, 5: 131-295, 1954. URL http://eudml .org/doc/73714.

A. Cousin and E. Di Bernardino. On multivariate extensions of Value-atRisk. Journal of Multivariate Analysis, 119:32-46, 2013. doi: 10.1016/j. jmva.2013.03.016. 
A. Cousin and E. Di Bernardino. On multivariate extensions of ConditionalTail-Expectation. Insurance: Mathematics and Economics, 55:272-282, 2014. doi: 10.1016/j.insmatheco.2014.11.006.

D. Denneberg. Distorted probabilities and insurance premiums. Methods of Operations Research, 63(3):3-5, 1990.

D. Denneberg. Non-Additive Measure and Integral. Springer, Dordrecht, 1994. doi: 10.1007/978-94-017-2434-0.

E. Di Bernardino and F. Palacios-Rodríguez. Estimation of extreme component-wise excess design realization: a hydrological application. Stochastic Environmental Research and Risk Assessment, Online:1-15, 2017. doi: 10.1007/s00477-017-1387-y.

P. Embrechts and G. Puccetti. Bounds for functions of multivariate risks. Journal of Multivariate Analysis, 97(2):526-547, 2006. doi: 10.1016/j. jmva.2005.04.001.

P. Embrechts, D.D. Lambrigger, and M. V. Wüthrich. Multivariate extremes and the aggregation of dependent risks: examples and counter-examples. Extremes, 12(2):107-127, 2009. doi: 10.1007/s10687-008-0071-5.

D. J. G. Farlie. The performance of some correlation coefficients for a general bivariate distribution. Biometrika, 47(3/4):307-323, 1960. doi: 10.1093/ biomet/47.3-4.307.

E. J. Gumbel. Bivariate exponential distributions. Journal of the American Statistical Association, 55(292):698-707, 1960. doi: 10.1080/01621459. 1960.10483368 .

INE, 2014. Spanish National Statistics Institute, http://www.ine.es/.

M.C. Jones and N. Balakrishnan. How are moments and moments of spacings related to distribution functions? Journal of Statistical Planning and Inference, 103(1):377-390, 2002. doi: 10.1016/S0378-3758(01)00232-4.

M.C. Jones, B.C. Arnold, H.A. David, J.T. Kent, H.N. Nagaraja, J.T.A.S. Ferreira, and M.F.J. Steel. Families of distributions arising from distributions of order statistics. Test, 13(1):1-43, 2004. doi: 10.1007/BF02602999. 
K.V. Mardia. Multivariate pareto distributions. The Annals of Mathematical Statistics, 33:1008-1015, 1962. doi: 10.1214/aoms/1177704468.

D. Morgenstern. Einfache beispiele zweidimensionaler verteilungen. Mitteilungsblatt für Mathematische Statistik, 8:234-235, 1956.

K. Pearson. Note on Francis Galton's problem. Biometrika, 1(4):390-399, 1902. doi: 10.2307/2331627. Published in Francis Galton's article.

L. Rüschendorf. Law invariant convex risk measures for portfolio vectors. Statistics \&3 Decisions, 24:97-108, 2006. doi: 10.1524/stnd.2006.24.1.97.

L. Rüschendorf. Mathematical Risk Analysis. Dependence, Risk Bounds, Optimal Allocations and Portfolios. Springer-Verlag, Berlin, 2013. ISBN 9783-642-33589.

G. Salvadori, C. De Michele, and F. Durante. On the return period and design in a multivariate framework. Hydrology and Earth System Sciences, 15:3293-3305, 2011. doi: 10.5194/hess-15-3293-2011.

E.W. Sun, Y.J. Wang, and M.T. Yu. Integrated portfolio risk measure: Estimation and asymptotics of multivariate geometric quantiles. Computational Economics, Online:1-26, 2017. doi: 10.1007/s10614-017-9708-2.

S. Wang. Insurance pricing and increased limits ratemaking by proportional hazards transforms. Insurance: Mathematics and Economics, 17(1):43-54, 1995a. doi: 10.1016/0167-6687(95)00010-P.

S. Wang. Premium calculation by transforming the layer premium density. Astin Bulletin, 26(1):71-92, 1995b. doi: 10.2143/AST.26.1.563234. 